\title{
Inhibitory effect of MyoD on the proliferation of breast cancer cells
}

\author{
CHANGJING CAI, XIAOQUN QIN, ZIYI WU, QIXIA SHEN, WENQIAN YANG, \\ SHUJUN ZHANG, JINLING DUAN, FENGLAN LIANG and CHI LIU
}

Physiology Department, Xiangya Medical School, Central South University, Changsha, Hunan 410078, P.R. China

Received January 11, 2015; Accepted February 24, 2016

DOI: $10.3892 / 01.2016 .4448$

\begin{abstract}
Skeletal muscle is rich in lymphatic vessels, with an abundant blood supply, and it is an infrequent site of cancer metastasis. Previous studies have demonstrated that enhanced secretion of MyoD may occur when skeletal muscle is injured or becomes cancerous. It was hypothesized that MyoD may act as an endogenous cytokine to inhibit the proliferation of cancer cells. To verify the possible effect of this protein on tumor cell proliferation, $\mathrm{C} 2 \mathrm{C} 12$ mouse skeletal muscle cells and 4T1 mouse breast cancer cells were co-cultured using embedded Transwell plates. Following co-culture, cell cycle analysis revealed that $\mathrm{C} 2 \mathrm{C} 12$ muscle cells were able to inhibit the proliferation of the breast cancer cells. Subsequently, MyoD was silenced in $\mathrm{C} 2 \mathrm{C} 12$ cells to assess its effect on $4 \mathrm{~T} 1$ cell proliferation. Following co-culture with $\mathrm{MyoD}$-silenced cells, a 5-ethynyl-20-deoxyuridine assay indicated that MyoD silencing prevented the reduction in proliferation of 4T1 cells induced by untransfected $\mathrm{C} 2 \mathrm{C} 12$ cells. In summary, the results indicated that $\mathrm{MyoD}$ inhibits the proliferation of breast cancer cells and may be a tumor suppressor factor.
\end{abstract}

\section{Introduction}

MyoD was first cloned in 1987 and termed MyoD1 (1). The protein has a basic helical three-dimensional crystal structure containing a basic helix-loop-helix domain that is able to bind to other proteins that also possess this domain, including myocyte-enhancing factor 2 , myogenin and creatine kinase (CK). Its adjacent basic region is required for it to bind to the promoters or enhancers of numerous muscle-specific genes, including CK and myogenin $(1,2)$. The N-terminus of MyoD contains a histidine-cysteine domain and a transcription activation domain, which are associated with the transcriptional activation of MyoD target genes, and the C-terminus contains

Correspondence to: Professor Chi Liu, Physiology Department, Xiangya Medical School, Central South University, 110 Xiangya Road, Changsha, Hunan 410078, P.R. China

E-mail: liuchi7669@gmail.com

Key words: skeletal muscle, metastatic cancer, MyoD, breast cancer a facultative helical (helix III) domain that may be associated with chromatin remodeling (3-5).

As a member of the muscle transcription factor family, MyoD has decisive roles in muscle differentiation, including muscle conversion and the maintenance of muscle differentiation $(1,2)$. Recent studies have demonstrated that a synthetic MyoD polypeptide has a high affinity for the inhibitor of DNA-binding proteins (ID), and thus may inhibit the binding of ID with DNA, thereby inhibiting the proliferation of cancer cells (6). In addition, Dey et al (7) identified that $\mathrm{MyoD}$ is an important cytokine in cerebellar development and a tumor suppressor gene in medulloblastoma. These previous studies strongly indicate the existence of a close association between MyoD and cancer cells.

As a major organ, skeletal muscle is rich in lymphatic vessels with an abundant blood supply. However, few studies have demonstrated cancer metastasis to skeletal muscle tissue (8-12). MyoD expression may be increased following skeletal muscle injury or its invasion by cancer cells $(13,14)$. The present study aimed to test the hypothesis that MyoD may act as an endogenous cytokine to inhibit the growth of metastatic cancer. Its expression was assessed in breast cancer tissue and cell lines and in $\mathrm{C} 2 \mathrm{C} 12$ skeletal muscle cells, and the proliferation of breast cancer cells was evaluated following co-culture with control or MyoD-silenced skeletal muscle cells.

\section{Materials and methods}

Cell culture and co-culture. The immortalized mouse myoblast cell line $\mathrm{C} 2 \mathrm{C} 12$ and the mouse breast tumor cell line 4T1 (each gifted by the Xiangya Central Experiment Laboratory, Changsha, China) were maintained at $37^{\circ} \mathrm{C}$ in an atmosphere of 5\% $\mathrm{CO}_{2}$ in Dulbecco's modified Eagle's medium (DMEM) supplemented with $100 \mathrm{U} / \mathrm{ml}$ penicillin, $100 \mathrm{U} / \mathrm{ml}$ streptomycin and $10 \%$ heat-inactivated fetal bovine serum (all purchased from Sigma-Aldrich China, Inc., Shanghai, China).

Transwell chambers $(0.4-\mu \mathrm{m}$ pore size; Corning Incorporated, Corning, NY, USA) were placed into 6-well plates. The interior of the Transwell plate was designated the upper chamber, while the space between the plates formed the lower chamber, and the chambers were separated by a polycarbonate membrane. Due to the permeability of the polycarbonate membranes, components in the lower-layer medium are able to affect the growth and movement of cells 
placed in the upper chamber. In order to study the impact of cytokines secreted by skeletal muscle cells on cancer cells, Transwell chambers were used to form a co-culture, with skeletal muscle cells in the lower chamber and cancer cells in the upper chamber (15). C1C12 and 4T1 cells were firstly cultured in a culture flask to at a cell concentration of $5 \times 10^{5} / \mathrm{ml}$ for $\sim 48$ h until they reached $70 \%$ confluence. The $\mathrm{C} 2 \mathrm{C} 12$ cells were subsequently transplanted onto a 6-well plate (Corning Incorporated) for $24 \mathrm{~h}$, and the $4 \mathrm{~T} 1$ cells were cultured in Transwell (Corning Incorporated). The cells were co-cultured for $48 \mathrm{~h}$ with the $4 \mathrm{~T} 1$ cells in the upper chambers and the $\mathrm{C} 1 \mathrm{C} 12$ cells in the lower chambers.

Immunohistochemical analysis. Breast cancer tissues and adjacent non-cancer tissues were obtained from 7 randomly selected patients diagnosed with breast cancer at the Xiangya Hospital of Central South University (Changsha, China). Breast cancer tissue was dissected away from normal tissue, fixed with $4 \%$ paraformaldehyde, embedded in paraffin and cut into $5-\mu \mathrm{m}$ sections. A primary mouse monoclonal anti-MyoD antibody (\#sc-32758; Santa Cruz Biotechnology, Inc., Santa Cruz, CA, USA) was used to detect MyoD expression. Briefly, endogenous peroxidase was inhibited by soaking tissue sections in $3 \% \mathrm{H}_{2} \mathrm{O}_{2}$. After rinsing in phosphate-buffered saline (PBS), sections were incubated with goat serum (Sigma-Aldrich China, Inc.) to block the non-specific binding of antibodies, and sections were then incubated overnight at $4-8^{\circ} \mathrm{C}$ with the anti-MyoD primary antibody (dilution, 1:50). After washing in PBS, the sections were incubated with biotinylated goat anti-rabbit IgG polyclonal antibody (dilution, 1:1,000; \#A6667; Sigma-Aldrich China, Inc.) for $1 \mathrm{~h}$ at room temperature and washed again. A streptavidin-biotin-peroxidase complex (\#RPN1051-2ML; GE Healthcare Life Sciences, Shanghai, China) was then incubated with the sections for $60 \mathrm{~min}$ at room temperature. After washing in PBS, the signal was detected with 3,3-diaminobenzidine. A negative control in which the primary antibody was omitted was included for each biopsy. Written informed consent was obtained from all patients and ethical approval was provided by the Medical Ethics Committee of the Basic Medical College of Central South University (Changsha, China).

Immunofluorescence. Sections were freed from the paraffin, rehydrated, subjected to antigen retrieval in $10 \mathrm{mM}$ sodium citrate, and treated with hydrogen peroxide. Sections were then blocked with 5\% goat serum containing 3\% Triton X-100 and incubated with the mouse monoclonal anti-MyoD antibody (dilution, 1:200) at $10 \mu \mathrm{g} / \mathrm{ml}$ for $1 \mathrm{~h}$ at room temperature. Next, slides were incubated with $\mathrm{ABC}$ reagent (from the VECTASTAIN ${ }^{\circledR}$ Elite ABC kit; Vector Laboratories, Inc., Burlingame, CA, USA) and Alexa Fluor 568-conjugated goat anti-mouse IgG (dilution, 1:1,000; \#A-11004; Thermo Fisher Scientific, Inc., Carlsbad, CA, USA), washed, and incubated with Tyramide Signal Amplification reagent (Thermo Fisher Scientific, Inc.) according to the manufacturer's instructions. Finally, the slides were washed and mounted using fluorescence mounting medium (Dako Omnis; Agilent Technologies, Santa Clara, CA, USA) containing $500 \mu \mathrm{g} / \mathrm{l}$ 4',6-diamidino-2-phenylindole for nuclei staining. Nuclei were then counterstained with $2 \%$ purified methyl green for $2 \mathrm{~min}$.
Table I. siRNA sequences and properties.

\begin{tabular}{lcc}
\hline siRNA & Sequences & GC\% \\
\hline 01 & 5'-GCCUGAGCAAAGUGAAUGA-dTdT-3' & 39 \\
& 3'-dTdT-CGGACUCGUUUCACUUACU-5' & 39 \\
02 & 5'-CAGCAGACGACUUCUAUGA-dTdT-3' & 39 \\
& 3'-dTdT-GUCGUCUGCUGAAGAUACU-5' & 39 \\
03 & 5'-CCAACUGCUCUGAUGGCAU-dTdT-3' & 43 \\
& 3'-dTdT-GGUUGACGAGACUACCGUA-5' & 43 \\
\hline
\end{tabular}

The slides incubated in the dark for $24 \mathrm{~h}$ prior to examination with a Zeiss LSM 510 laser-scanning confocal microscope (Zeiss GmbH, Jena, Germany).

MyoD staining intensity was determined using a color video camera (Sony DXC-950P; Sony Corporation, Tokyo, Japan) connected to a Leica Q500 IW Imaging Workstation with MoticFluo software v1.0 (Leica Microsystems, Cambridge, UK). A semi-quantitative scoring method was employed by three independent observers who were blinded to the conditions in order to record MyoD staining expression; scores were assigned on a scale of $0-4$ ( 0 , no staining; 4 , maximum staining) according to the staining intensities.

Small interfering RNA (siRNA) synthesis and transfection. Candidate siRNAs directed against MyoD mRNA were designed by Guangzhou RiboBio Co., Ltd. (Guangzhou, China). Three potential siRNAs were selected corresponding with the prediction of single-strand domains within the mRNA secondary structure (Table I). BLAST analyses (http://blast. ncbi.nlm.nih.gov/Blast.cgi) were performed to ensure that no additional significantly matching mouse transcripts would be targeted by these siRNAs. MyoD and nonsense siRNAs were synthesized by Guangzhou RiboBio Co., Ltd., and transfections were conducted with Invitrogen Lipofectamine 2000 (Thermo Fisher Scientific, Inc.), according to the manufacturer's instructions. Reverse transcription (RT)-polymerase chain reaction (PCR) and western blotting were used to detect MyoD expression following siRNA transfection.

Semi-quantitative RT-PCR. The sequences of the primers for mouse MyoD were as follows: MyoD forward, 5'-CTC CTTTGAGACAGCAGACGACTT-3', and reverse, 5'-AAA TCGCATTGGGGTTTGAGCCTG-3'; and $\beta$-actin forward, 5'-GAAACTACCTTCAACTCCATC-3', and reverse, 5'-CGA GGCCAGGATGGAGCCGCC-3'. Primers were designed by Primer-BLAST (www.ncbi.nlm.nih.gov/tools/primer-blast/). $\beta$-actin was used to ascertain the presence of an equal amount of cDNA in each reaction. A TRIzol ${ }^{\circledR}$ kit (Thermo Fisher Scientific, Inc.) was used to extract genomic RNA. Total RNA $(1 \mu \mathrm{g})$ purified from siRNA-transfected cells was reverse transcribed into cDNA using AMV reverse transcriptase (Qiagen, Venlo, Netherlands) with an RNase inhibitor (Thermo Fisher Scientific, Inc.) and oligo(dT) primer (Thermo Fisher Scientific, Inc.) at $40^{\circ} \mathrm{C}$ for $50 \mathrm{~min}$, followed by heating at $90^{\circ} \mathrm{C}$ for $5 \mathrm{~min}$. Next, $1 \mu \mathrm{l}$ reverse-transcriptase was added to a $30 \mu \mathrm{l}$ PCR mixture [Bio-Rad Laboratories (Singapore) Pte. Ltd., Singapore] for 30 cycles. Taq polymerase was added 
from the SuperScript ${ }^{\circledR}$ III One-Step RT-PCR system (Thermo Fisher Scientific, Inc.), and each PCR cycle consisted of $93^{\circ} \mathrm{C}$ for $30 \mathrm{sec}$ and $54^{\circ} \mathrm{C}$ for $60 \mathrm{sec}$. Negative controls consisted of an equal volume of water substituted for the volume of RNA in the RT reaction. mRNA expression data for sample-to-sample variability in RNA input, RNA quality and reverse transcription efficiency was normalized to $\beta$-actin.

Western blotting. MyoD protein expression was detected by western blotting using the aforementioned MyoD monoclonal antibody. For the preparation of cell extracts, cells from different groups were washed three times with ice-cold PBS and then lysed in lysis buffer [50 mM Tris- $\mathrm{HCl}(\mathrm{pH} 8.0)$, $150 \mathrm{mM} \mathrm{NaCl}, 1 \mathrm{mM}$ ethylenediaminetetraacetic acid, $1 \%$ Triton X-100 and $100 \mu \mathrm{g} / \mathrm{ml}$ phenylmethylsulfonyl fluoride] on ice for $20 \mathrm{~min}$. Following centrifugation at $16,000 \mathrm{x} \mathrm{g}$ for 2 min at $4^{\circ} \mathrm{C}$, supernatants were separated using $10 \%$ sodium dodecyl sulfate-polyacrylamide gel electrophoresis, and then electrophoretically transferred to nitrocellulose membranes (Invitrogen; Thermo Fisher Scientific, Inc.). After blocking for $2 \mathrm{~h}$ with $5 \%$ fat-free milk at room temperature, the membranes were incubated with the primary mouse monoclonal anti-MyoD antibody or control mouse monoclonal anti- $\beta$-actin secondary antibody (dilution, 1:1,000; \#A1978; Sigma-Aldrich China, Inc.) for $24 \mathrm{~h}$ at $4^{\circ} \mathrm{C}$. The membranes were then incubated with a secondary biotinylated goat anti rabbit IgG polyclonal antibody (dilution, 1:1,000; \#A6667; Sigma-Aldrich China, Inc.) for $1 \mathrm{~h}$ at room temperature. Protein bands were visualized using Pierce enhanced chemiluminescence reagent (Thermo Fisher Scientific, Inc.) and Odyssey v1.2 software (LI-COR Biosciences, Lincoln, NE, USA). The intensity of expression was measured by comparing the target and control bands.

Cell cycle analysis using propidium iodide (PI) and flow cytometry. Cell cycle analysis was conducted at $72 \mathrm{~h}$ after transfection. $4 \mathrm{~T} 1$ cells $\left(5 \times 10^{5}\right)$ from the tested groups were harvested by brief trypsinization, washed twice with PBS, fixed in $70 \%$ ethanol overnight and stained with PI (final concentration, $20 \mathrm{mg} / \mathrm{ml}$ )/Triton X-100 solution containing $10 \mathrm{mg} / \mathrm{ml}$ RNase (DNase-free (Thermo Fisher Scientific, Inc.). Following incubation at $37^{\circ} \mathrm{C}$ for $30 \mathrm{~min}$, the samples were analyzed using a FACSCalibur flow cytometer (BD Biosciences, Franklin Lakes, NJ, USA), and the populations of cells in G1, S and G2 were quantified. The BD FACSDiva software (BD Biosciences) can export data files in FCS 2.0 or 3.0 default formats. The ModFit LT v3.0 software package (Verity Software House, Topsham, ME, USA) was used. The Click-iT® EdU Alexa Fluor ${ }^{\circledR} 488$ Imaging Kit (Thermo Fisher Scientific, Inc.) is a superior alternative to traditional proliferation assays that is optimized for fluorescence microscopy applications.

5-ethynyl-20-deoxyuridine (EdU) assay. EdU is a nucleoside analog of thymidine that is incorporated into DNA during active DNA synthesis by proliferating cells, and may be visualized by the addition of a fluorescent molecule. Thus, proliferating 4T1 cells were detected using a Cell-Light ${ }^{\mathrm{TM}}$ EdU Apollo ${ }^{\circledR} 567$ in vitro Imaging Kit (Guangzhou RiboBio Co., Ltd.) according to the manufacturer's protocol. Briefly, cells were incubated with $500 \mu \mathrm{l}$ of $50 \mu \mathrm{M}$ EdU for $3 \mathrm{~h}$ before fixation, permeabilization and visualization of EdU staining. Cell nuclei were stained with $10 \mathrm{mg} / \mathrm{ml}$ Hoechst 33342 (Guangzhou RiboBio Co., Ltd) for $30 \mathrm{~min}$. Quantification of the staining intensity was determined using a color video camera (DXC-950P; Sony, Tokyo, Japan). The camera was connected to a Leica Imaging Workstation with MoticFluo 1.0 imaging software (Leica Q500IW; Leica, Cambridge, UK).

Statistical analysis. All experiments were performed at least five times. All data are presented as the mean \pm standard error of the mean. For all determinations, the differences were considered significant when $\mathrm{P}<0.05$. The unpaired $t$-test was used for comparing two groups. All statistical analyses were performed using SPSS software version 18 (SPSS, Inc., Chicago, IL, USA).

\section{Results}

Proliferation of $4 T 1$ cells may be suppressed by C2C12 cells. To identify whether skeletal muscle cells can inhibit the proliferation of cancer cells, mouse breast tumor cells (4T1 cells) and mouse myoblast cells (C2C12 cells) were co-cultured on Transwell plates. Mouse breast tumor cells and mouse breast tumors were co-cultured as controls. PI staining and flow cytometry were used to detect the proliferation of the 4T1 cells. As shown in Fig. 1, at $48 \mathrm{~h}$ after co-culture, $65 \%$ of the cells were in $\mathrm{G} 1$ phase and $33 \%$ were in S phase in the experimental group, compared to $31 \%$ in G1 phase and $56 \%$ that were in $\mathrm{S}$ phase in the control group $(\mathrm{G} 1: \mathrm{P}=0.0376$ vs. control group; $\mathrm{S}$ : $\mathrm{P}=0.0396$ vs. control group; $\mathrm{G} 2 / \mathrm{M}$ : $\mathrm{P}=0.0479$ vs. control group; $n=6$ ), demonstrating that the proliferation of the 4T1 cells was inhibited following co-culture with the $\mathrm{C} 2 \mathrm{C} 12$ cells (Fig. 1A and B).

Silencing efficiency of MyoD siRNAs. RNA interference was used to generate a $\mathrm{C} 2 \mathrm{C} 12$ cell line with targeted silencing of MyoD. To verify the silencing efficiency of MyoD siRNA, three candidate siRNAs were designed and numbered 01, 02 and 03, respectively. The expression of MyoD mRNA in C2C12 cells was detected using RT-PCR following siRNA transfection at the recommended concentration of $100 \mathrm{nM}$. As shown in Fig. 2A and B, all three siRNAs effectively downregulated MyoD expression in the $\mathrm{C} 2 \mathrm{C} 12$ cells $(\mathrm{P}=0.00134$ vs. control siRNA; $n=3)$, and the silencing efficiency of siRNA 01 reached $\sim 70 \%$. Therefore siRNA 01 was selected for use in subsequent experiments.

siRNA 01 was transfected into $\mathrm{C} 2 \mathrm{C} 12$ cells using Lipofectamine 2000 and western blotting was performed to investigate MyoD protein expression at $72 \mathrm{~h}$ after transfection (Fig. 2C and D). The results demonstrated that the expression of MyoD was markedly reduced compared with the control group, which consisted of untransfected cells. Gray value analysis indicated that MyoD expression in the experimental group was decreased by $\sim 70 \%$ compared with the control group ( $\mathrm{P}=0.00149$ vs. control siRNA; $n=3$ ), which was consistent with the RT-PCR results, and the nonsense siRNA had no effect on MyoD expression.

Proliferation of $4 T 1$ cells is inhibited by MyoD. To explore the effects of MyoD and other cytokines from skeletal muscle 
A

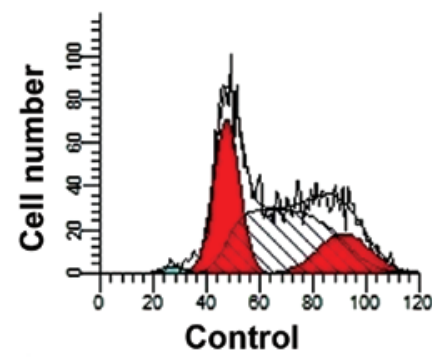

B

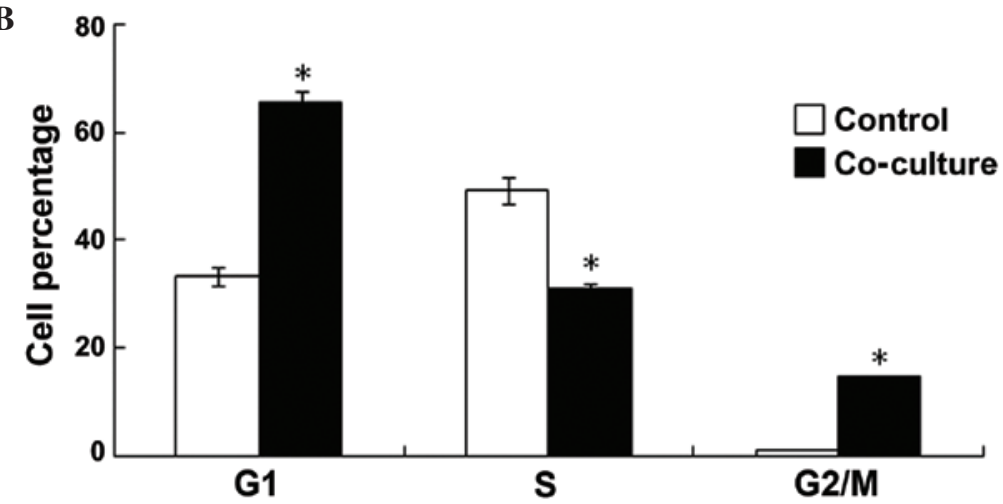

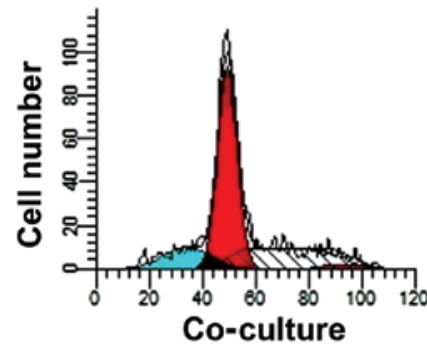

Figure 1. Proliferation of 4T1 cells was suppressed by co-culture with $\mathrm{C} 2 \mathrm{C} 12$ skeletal muscle cells. (A) After $48 \mathrm{~h}$ of co-culture, the populations of cells in G1, S and G2/M phases from the different groups were separated and counted. (B) Cell populations in the G1, S and G2/M phases are shown in histograms produced using ModFit LT v3.0 software package. " $\mathrm{P}<0.05$ vs. control (4T1 cells cultured in the absence of C2C12 cells).

A

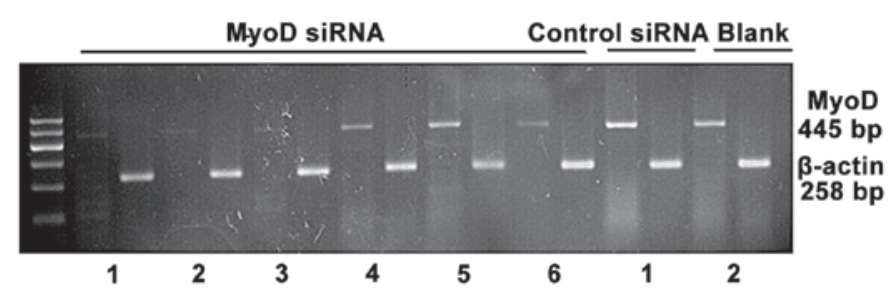

C

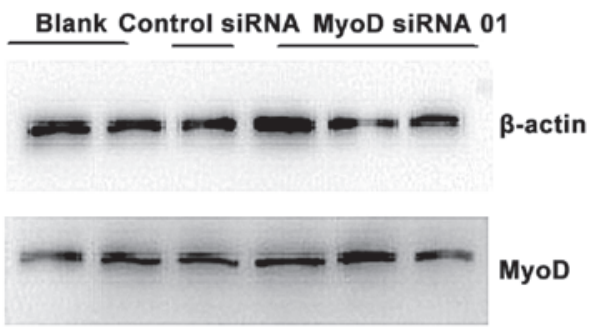


A
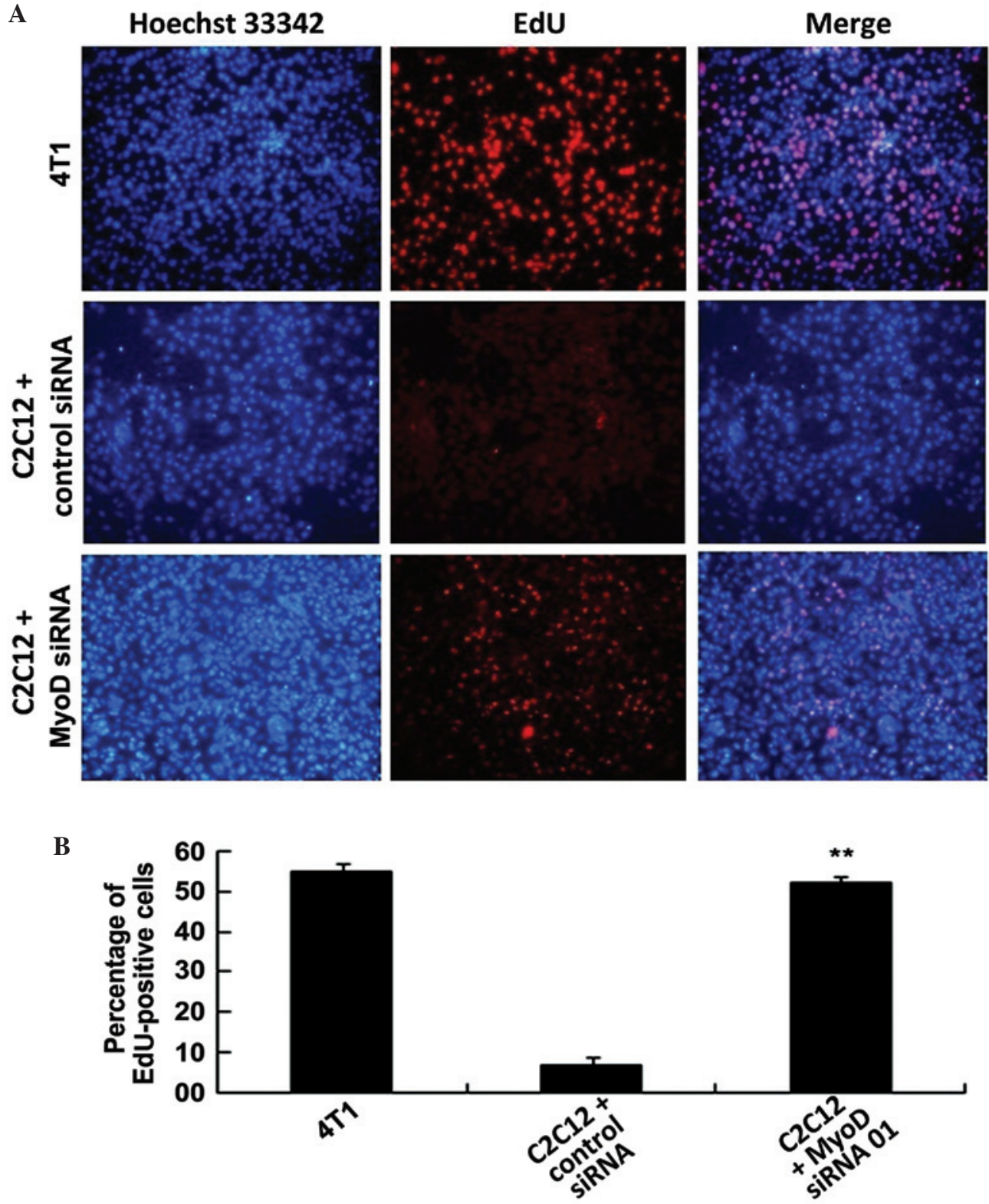

Figure 3. Proliferation of 4T1 cells can be inhibited by MyoD. (A) 4T1 cells were labeled with EdU following co-culture with MyoD siRNA-transfected or control siRNA-transfected C2C12 cells, or with only $4 \mathrm{~T} 1$ cells, in Transwell chambers. The Click-iT reaction revealed EdU staining (red), and cell nuclei were stained with Hoechst 33342 (blue). The images are representative of the results obtained. (B) The percentage of EdU-positive 4T1 cells was quantified. The data are presented as the mean \pm standard error of the mean. ${ }^{* *} \mathrm{P}<0.01$ vs. $4 \mathrm{~T} 1$.

was extremely sparse and was detected in $\sim 10 \%$ of all cells (untransfected $\mathrm{C} 2 \mathrm{C} 12$ vs. $4 \mathrm{~T} 1, \mathrm{P}=0.00648 ; \mathrm{n}=3$ ). However, the EdU fluorescence of the 4T1 cells co-cultured with the C2C12 cells that had been transfected with MyoD siRNA was moderately bright, accounting for $\sim 40 \%$ of all cells with gray signals (C2C12+MyoD siRNA vs. $4 \mathrm{~T} 1, \mathrm{P}=0.00130 ; \mathrm{n}=3$ ). The proliferation of the $4 \mathrm{~T} 1$ cells co-cultured with the $\mathrm{C} 2 \mathrm{C} 12$ cells transfected with nonsense siRNA was similar to that of the 4T1 cells co-cultured with the untransfected $\mathrm{C} 2 \mathrm{C} 12$ cells (C2C12+control siRNA vs. 4T1, $\mathrm{P}=0.00539 ; \mathrm{n}=3$ ). These results indicate that $\mathrm{MyoD}$ is able to markedly inhibit the proliferation of the $4 \mathrm{~T} 1$ cells.

MyoD is weakly expressed in $4 T 1$ and human breast cancer cells. To detect whether cancer cells express MyoD, TRIzol was used to extract genomic RNA and total protein from 4T1 cells after $48 \mathrm{~h}$ of culturing in 6-well plates and then performed RT-PCR and western blotting. Only faint bands were observed by RT-PCR ( $\mathrm{P}=0.00278$ vs. $\beta$-actin; $\mathrm{n}=3)$ and western blotting $(\mathrm{P}=0.00324$ vs. $\beta$-actin; $\mathrm{n}=3)$, indicating weak MyoD expression in the 4T1 cells (Fig. 4A-D).

To further characterize MyoD expression in breast cancer tissue, breast cancer and normal tissue samples were obtained from 7 randomly selected breast cancer patients and assessed its expression using immunofluorescence and immunohistochemistry. The two assays revealed weak MyoD expression in the breast cancer and control human breast tissues (immunofluorescence: $0.97 \pm 0.01, \mathrm{P}=0.00448$ vs. control, $\mathrm{n}=7$, unpaired $t$-test; immunohistochemistry: $0.95 \pm 0.01$, $\mathrm{P}=0.00377$ vs. control, $\mathrm{n}=7$, unpaired $t$-test) (Fig. 4E-H). In addition, Fig. 4 shows evident differences between the cancer and control tissues. The present study aimed to test 
A

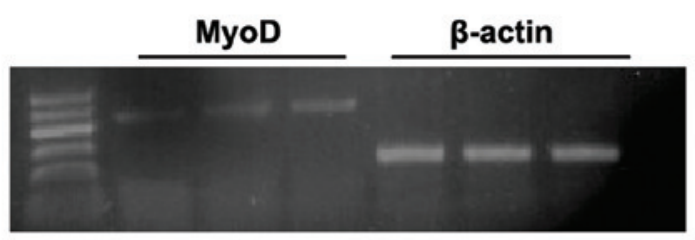

C
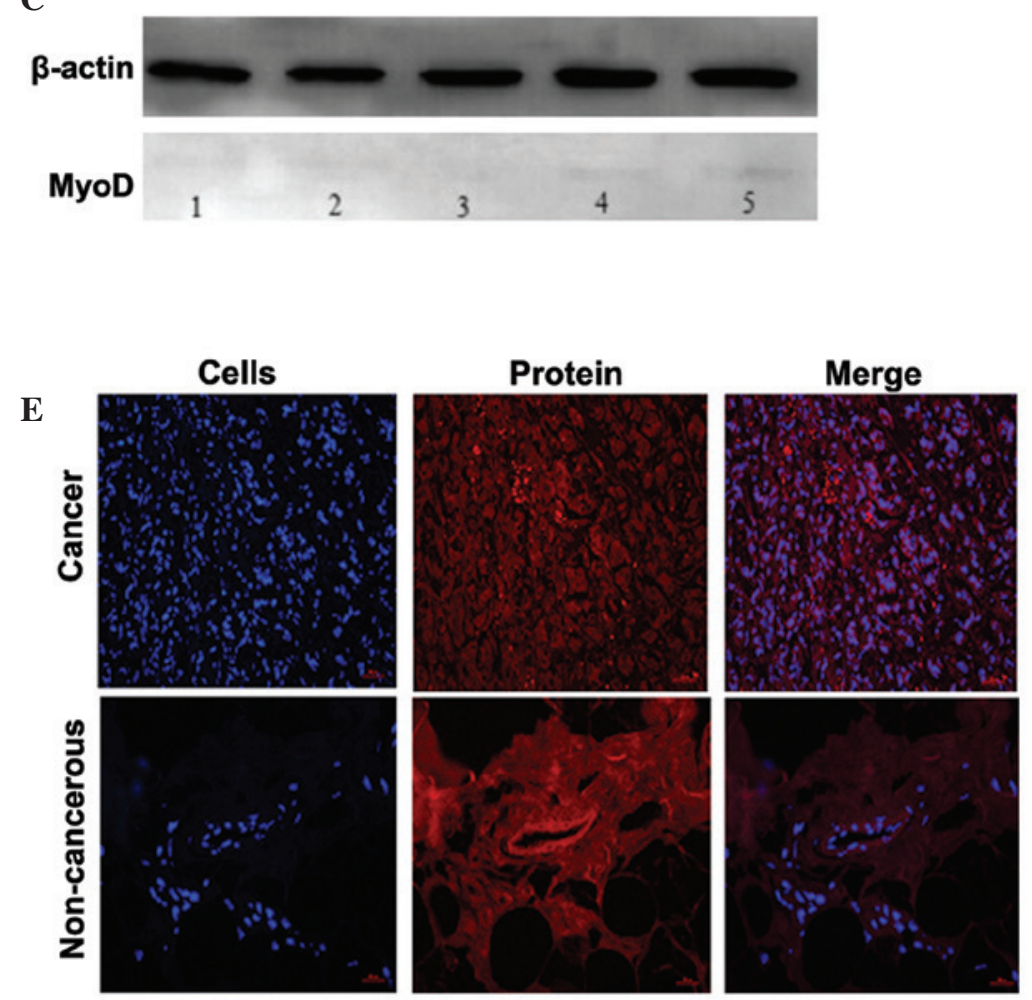

G

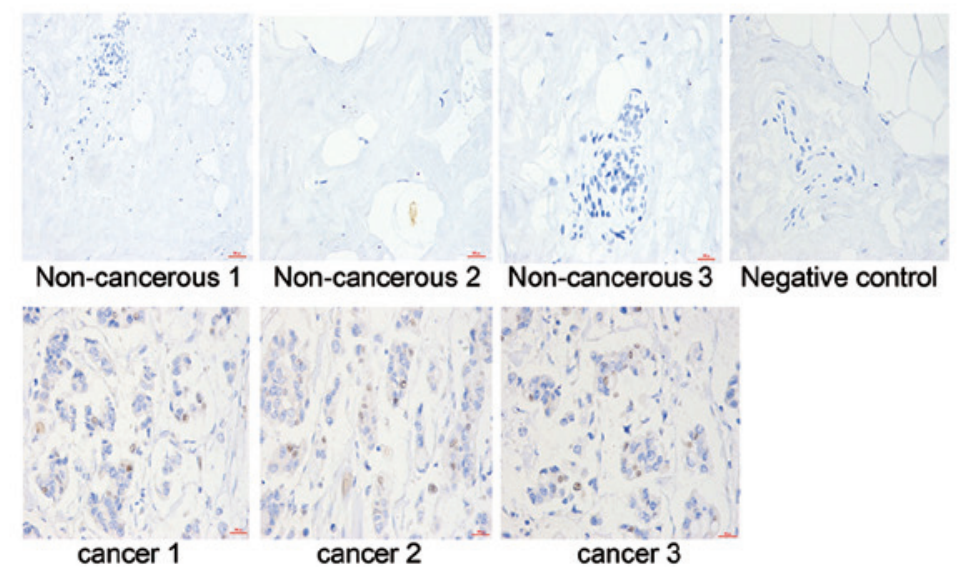

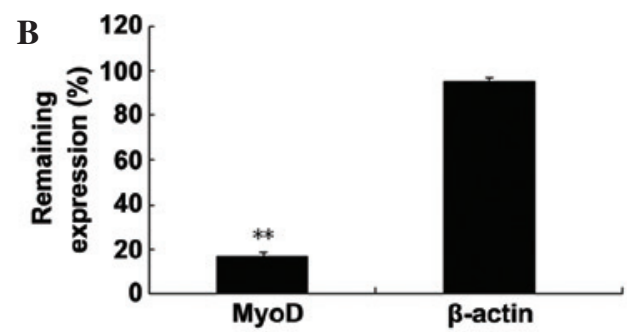

D

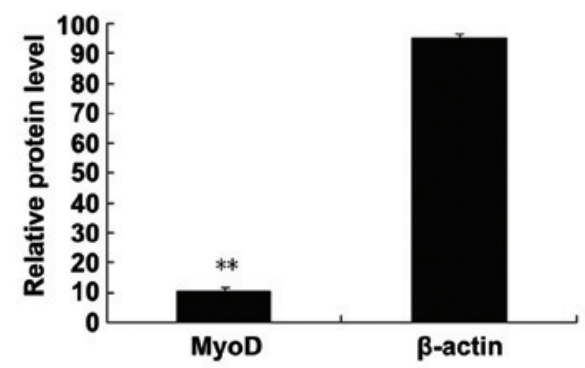

F

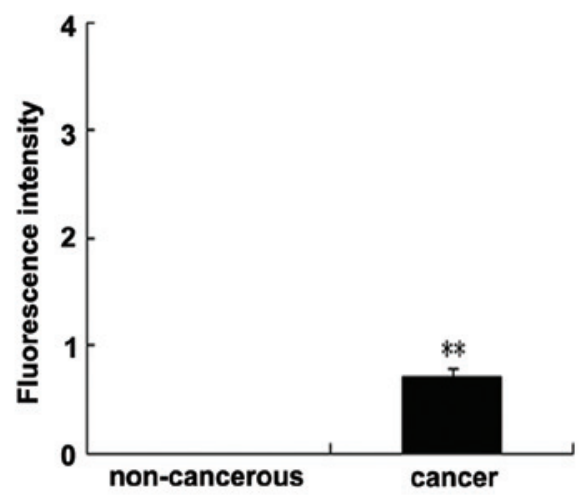

$\mathbf{H}$

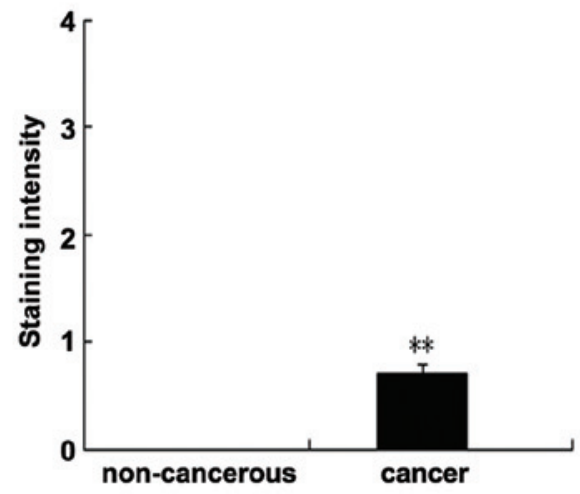

Figure 4. MyoD was weakly expressed in breast cancer tissues. (A) Results of reverse transcription-polymerase chain reaction analysis of MyoD mRNA expression and (B) semi-quantification relative to $\beta$-actin ( ${ }^{* *} \mathrm{P}<0.01$ vs. $\beta$-actin). (C) Results of western blot analysis of MyoD protein expression and (D) quantification relative to $\beta$-actin $\left({ }^{* *} \mathrm{P}<0.01\right.$ vs. $\beta$-actin). (E) Representative MyoD expression in human breast cancer and control tissues as revealed by immunofluorescence and (F) semi-quantitative scores $\left({ }^{* *} \mathrm{P}<0.01\right.$ vs. non-cancerous). (G) Representative MyoD expression in human breast cancer and control tissues as revealed by immunohistochemistry and $(\mathrm{H})$ semi-quantitative scores $\left({ }^{* *} \mathrm{P}<0.01\right.$ vs. non-cancerous $)$.

the expression of MyoD in the tissues. The other differences between the control tissues and the cancer may be due to the atypia of cancer cells. To the best of our knowledge, this is the first report of low levels of MyoD expression in breast cancer tissue.

\section{Discussion}

Skeletal muscle is widely distributed and is an infrequent site of cancer metastasis (9). The current study was conducted to test the hypothesis that an endogenous tumor suppressor factor 
may be associated with the low incidence of cancer metastasis in skeletal muscle. MyoD secretion increases when skeletal muscle is injured or invaded by cancer cells, suggesting that there is an association between MyoD and tissue wound repair (1). We hypothesized that MyoD may be an endogenous tumor suppressor factor that is also associated with the low occurrence of cancer in skeletal muscle (16).

MyoD is a DNA-binding protein that also has a significant role in skeletal muscle differentiation due to its importance in muscle conversion (6). Recently, Dey et al (7) demonstrated that MyoD is an important cytokine during cerebellar development and is a tumor suppressor gene in medulloblastoma. In fact, MyoD may regulate gene expression as a DNA-binding protein. Chen et al (6) used synthetic peptide fragments of MyoD to block the binding of DNA with ID, which is an important regulator of cell proliferation. After ID binding is blocked, cancer cell proliferation decreases (17), indicating a possible pathway by which MyoD may inhibit the proliferation of these cells. However, MyoD is a large protein, and its ability to enter the cell and affect DNA duplication require further verification $(18,19)$.

As it is difficult to monitor the biological activity of MyoD in vitro, a MyoD-silenced model of mouse myoblast $\mathrm{C} 2 \mathrm{C} 12$ cells was constructed in the present study. The $\mathrm{C} 2 \mathrm{C} 12$ cells were co-cultured with $4 \mathrm{~T} 1$ mouse breast cancer cells in Transwell chambers to explore the effects of MyoD on the proliferation of cancer cells. PI and EdU assays were used to assess cancer cell proliferation and the results revealed that the proliferation of the 4T1 cells was markedly inhibited by the $\mathrm{C} 2 \mathrm{C} 12$ cells. PI staining results revealed that the population of cancer cells in S phase was $20 \%$ lower following co-culture with skeletal muscle cells, and the population in G1 phase was $35 \%$ higher than that of the control group. These results indicate that skeletal muscle cells may inhibit cancer cell proliferation by regulating the cell cycle.

To further assess the effects of MyoD on cancer cell proliferation, mouse breast tumor cells were co-cultured with MyoD-silenced mouse myoblast cells. Proliferation of the 4T1 cancer cells was significantly inhibited in the group that was co-cultured with the control (untransfected) $\mathrm{C} 2 \mathrm{C} 12$ cells. However, in the group that was co-cultured with the MyoD-silenced C2C12 cells, the 4T1 cells exhibited no change in proliferative activity compared with the 4T1 cells cultured in the absence of $\mathrm{C} 2 \mathrm{C} 12$ cells. These results suggest that MyoD was responsible for inhibiting the proliferation of the 4T1 cells, indicating that this protein may act as an endogenous factor to inhibit cancer cell proliferation (20).

The current study also evaluated MyoD expression in 4T1 cells by RT-PCR and western blotting. MyoD protein was found to be expressed at low levels in the 4T1 cells. In addition, its expression was assessed in the control human breast tissue and breast cancer tissue samples obtained from randomly selected breast cancer patients; no obvious MyoD expression was observed in the non-cancerous tissues, whereas this protein was expressed in the breast cancer tissue. Similarly to other tumor suppressor genes that are expressed only in cancer cells, such as $\alpha$-fetoprotein (21), MyoD was expressed in the cancer cells at levels insufficient to inhibit their proliferation. A recent study performed by Dey et al (7) has shown that MyoD is a tumor suppressor gene in medulloblastoma. The current results indicated that MyoD may act as a tumor suppressor gene in 4T1 cells; however, this conclusion requires further verification. In addition, the low incidence of skeletal muscle metastasis is not limited to one type of cancer cell, suggesting that MyoD may be a suppressor of multiple types of cancer $(8,9)$.

MyoD also serves important roles in muscle transformation in the skeletal muscle microenvironment. Several previous studies have reported the successful transformation of fat cells into skeletal muscle cells by MyoD transfection in vitro (22), and this technique has been widely applied in chicken, pork and beef production (23-25). Skeletal muscle spends a long period of time undergoing tissue differentiation, maturation and repair $(26,27)$. Cancer cells are a class of cells with high proliferative abilities (28-30). We suspect that it may also be possible to transform cancer cells into normal muscle tissue by taking advantage of the muscle conversion function of MyoD, the accomplishment of which would represent progress in cancer research.

In conclusion, the current study demonstrates for the first time that MyoD plays a critical role in cancer development by inhibiting the proliferation of cancer cells. Furthermore, it may act as a tumor suppressor gene in multiple types of cancer cells. These results will aid in the elucidation of the mechanisms underlying the low incidence of cancer metastasis in skeletal muscle.

\section{Acknowledgements}

The authors would like to thank the Department of Pathology of Xiangya Hospital for their support in obtaining the specimens. In addition, this work was supported by NSFC (grant nos. 81170016, 81270065, 81370116 and 81570026), Hunan Natural Science Foundation and National Basic Research Program of China (973 Program) (grant nos. 2013JJ4030, 2015JJ2147 and 2012CB518104), Youth Support Program of China Science Communication (grant no. 2015[45]) and National Innovative Entrepreneurship Training Program funded projects to University Students of Central South University (grant no. 201410533096).

\section{References}

1. Weintraub H, Davis R, Tapscott S, Thayer M, Krause M, Benezra R, Blackwell TK, Turner D, Rupp R and Hollenberg S: The MyoD gene family: Nodal point during specification of the muscle cell lineage. Science 251: 761-766, 1991.

2. Hirai H, Tani $\mathrm{T}$ and Kikyo N: Structure and functions of powerful transactivators: VP16, MyoD and FoxA. Int J Dev Biol 54: 1589-1596, 2010.

3. Asakura A, Lyons GE and Tapscott SJ: The regulation of MyoD gene expression: Conserved elements mediate expression in embryonic axial muscle. Dev Biol 171: 386-398, 1995.

4. Ishibashi J, Perry RL, Asakura A and Rudnicki MA: MyoD induces myogenic differentiation through cooperation of its NH2-and COOH-terminal regions. J Cell Biol 171: 471-482, 2005.

5. L'honoré A, Commère PH, Ouimette JF, Montarras D, Drouin J and Buckingham M: Redox regulation by Pitx 2 and Pitx 3 is critical for fetal myogenesis. Dev Cell 29: 392-405, 2014.

6. Chen CH, Kuo SC, Huang LJ, Hsu MH and Lung FD: Affinity of synthetic peptide fragments of MyoD for Id1 protein and their biological effects in several cancer cells. J Pept Sci 16: 231-241, 2010.

7. Dey J, Dubuc AM, Pedro KD, Thirstrup D, Mecham B, Northcott PA, Wu X, Shih D, Tapscott SJ, LeBlanc M, et al: MyoD is a tumor suppressor gene in medulloblastoma. Cancer Res 73: 6828-6837, 2013. 
8. Herrera L: Skeletal muscle metastases. Dis Colon Rectum 31: $579,1988$.

9. Surov A: Skeletal muscle metastases. Jpn J Radiol 32: 308-309, 2014.

10. Waning DL and Guise TA: Molecular mechanisms of bone metastasis and associated muscle weakness. Clin Cancer Res 20 3071-3077, 2014.

11. Carey K, Bestic J, Attia S, Cortese C and Jain M: Diffuse skeletal muscle metastases from sacral chordoma. Skeletal Radiol 43 985-989, 2014.

12. Gupta P, Gill M, Gupta S, Sachdeva B, Sansanwal P and Sen R: Metastatic Carcinoma in Skeletal Muscle-A Rare Presentation. Int J Health Sci Res (IJHSR) 4: 347-351, 2014.

13. Hatade T, Takeuchi K, Fujita N, Arakawa T and Miki A: Effect of heat stress soon after muscle injury on the expression of MyoD and myogenin during regeneration process. J Musculoskelet Neuronal Interact 14: 325-333, 2014.

14. Szuhai K, de Jong D, Leung WY, Fletcher CD and Hogendoorn PC: Transactivating mutation of the MYOD1 gene is a frequent event in adult spindle cell rhabdomyosarcoma. J Pathol 232: 300-307, 2014.

15. Demircan PC, Sariboyaci AE, Unal ZS, Gacar G, Subasi C and Karaoz E: Immunoregulatory effects of human dental pulp-derived stem cells on T cells: Comparison of transwell co-culture and mixed lymphocyte reaction systems. Cytotherapy 13: 1205-1220, 2011.

16. Battistelli C, Busanello A and Maione R: Functional interplay between $\mathrm{MyoD}$ and $\mathrm{CTCF}$ in regulating long-range chromatin interactions during differentiation. J Cell Sci 127: 3757-3767, 2014.

17. Lyden D, Young AZ, Zagzag D, Yan W, Gerald W, O'Reilly R, Bader BL, Hynes RO, Zhuang Y, Manova K and Benezra R: Id 1 and Id 3 are required for neurogenesis, angiogenesis and vascularization of tumour xenografts. Nature 401: 670-677, 1999

18. Berks BC, Lea SM and Stansfeld PJ: Structural biology of Tat protein transport. Curr Opin Struct Biol 27: 32-37, 2014.

19. Sorrentino V, Pepperkok R, Davis RL, Ansorge W and Philipson L: Cell proliferation inhibited by MyoD1 independently of myogenic differentiation. Nature 345: 813-815, 1990.
20. Rajabi HN, Takahashi C and Ewen ME: Retinoblastoma protein and $\mathrm{MyoD}$ function together to effect the repression of Fra-1 and in turn cyclin D1 during terminal cell cycle arrest associated with myogenesis. J Biol Chem 289: 23417-23427, 2014.

21. Gillespie JR and Uversky VN: Structure and function of alpha-fetoprotein: A biophysical overview. Biochim Biophys Acta 1480: 41-56, 2000.

22. Uezumi A, Fukada S, Yamamoto N, Takeda S and Tsuchida K Mesenchymal progenitors distinct from satellite cells contribute to ectopic fat cell formation in skeletal muscle. Nat Cell Biol 12: 143-152, 2010.

23. Cazzato D, Assi E, Moscheni C, Brunelli S, De Palma C, Cervia D, Perrotta $C$ and Clementi E: Nitric oxide drives embryonic myogenesis in chicken through the upregulation of myogenic differentiation factors. Exp Cell Res 320: 269-280, 2014.

24. Pena RN, Quintanilla R, Manunza A, Gallardo D, Casellas J and Amills M: Application of the microarray technology to the transcriptional analysis of muscle phenotypes in pigs. Anim Genet 45: 311-321, 2014

25. Zhang Q, Lee HG, Kang SK, Baik M and Choi YJ: Heat-shock protein beta 1 regulates androgen-mediated bovine myogenesis Biotechnol Lett 36: 1225-1231, 2014

26. Li VC and Kirschner MW: Molecular ties between the cell cycle and differentiation in embryonic stem cells. Proc Natl Acad Sci USA 111: 9503-9508, 2014.

27. Polesskaya A, Cuvellier S, Naguibneva I, Duquet A, Moss EG and Harel-Bellan A: Lin-28 binds IGF-2 mRNA and participates in skeletal myogenesis by increasing translation efficiency. Genes Dev 21: 1125-1138, 2007.

28. Lasorella A, Benezra R and Iavarone A: The ID proteins: Master regulators of cancer stem cells and tumour aggressiveness. Nat Rev Cancer 14: 77-91, 2014.

29. Emlet DR, Gupta P, Holgado-Madruga M, Del Vecchio CA, Mitra SS, Han SY, Li G, Jensen KC, Vogel H, Xu LW, et al: Targeting a glioblastoma cancer stem-cell population defined by EGF receptor variant III. Cancer Res 74: 1238-1249, 2014.

30. de Mendoza FH and Alanya Rodriguez E: Cancer Stem Cells in Brain Tumors. In: Stem Cells in Cancer: Should We Believe or Not? Grande E and Antón Aparicio L (eds). Springer, Netherlands, pp229-243, 2014. 